\title{
Formatos para preparación e informe de histeroscopia
}

\author{
Alba Lucía Mondragón de Villarreal*
}

RESUMEN: EI desarrollo de los procedimientos endoscópicos, ha hecho de la histeroscopia, un examen cada día más frecuente. En este artículo se realiza un análisis de los formatos educativos, utilizados previamente al procedimiento, los consentimientos o autorizaciones y los informes de los hallazgos encontrados. Se presentan tres modelos, con el fin de difundirlos y de unificar, el informe de histeroscopia diagnóstica, en este momento, en el cual, este método diagnóstico, sé esta generalizando, permitiendo futuros análisis comparativos, entre diferentes regiones del país.

PALABRAS CLAVES. Histeroscopia, endoscopia ginecológica, informe, documentación, educación, consentimiento.

SUMMARY: The development of the procedures endoscopy, has made of the histeroscopy a procedure every day but it frequents. I am carried out an analysis of the educational form, used previously to the procedure, the consents form or authorizations and the documentation form, for reports of the opposing discoveries. Three models are presented with the purpose of diffusing them and of unifying the histeroscopy report it diagnoses, at this time, in the one which, this method diagnoses I know this generalizing, allowing future comparative analysis in different regions of the country.

KEY WORDS: Histeroscopy, gynecological endoscopy, documentation form, education form, consent form.

\section{Introducción}

La histeroscopia, no es un procedimiento diagnóstico nuevo. Fue realizado satisfactoriamente desde 1869 , cuando Pantaleoni, observó por primera vez la cavidad uterina humana, con un tubo y una luz externa (1); pero solamente en los últimos años, se ha generalizado su uso y es una realidad en nuestro país, que muchos institutos y clínicas la utilizan de acuerdo a los protocolos internacionales, y que centros de educación, cada día están mas motivados, para que los residentes adquieran destreza en esta tecnología.

Quiero analizar este método diagnóstico, desde tres puntos de vista: primero: la preparación, el material educativo o la información que se le ofrece a la paciente, antes del procedimiento; segundo: el tipo de autorización o consentimiento que firman o que otorgan a los médicos que van a realizar la histeroscopia y tercero: el formato del informe de los hallazgos encontrados durante la histeroscopia.

En este artículo, presento tanto el análisis de los formatos preexistentes como los formatos que he diseñado y que utilizo en mi centro.

\section{Preparación}

Está contemplado en el código de Etica Médica (2) la importancia de entregar una información muy detallada de cada procedimiento médico a realizar, incluyendo los riesgos.
En la mayoría de las instituciones, este tipo de información es proporcionada por el médico tratante, en forma verbal y ocasionalmente, por personal paramédico o en entrenamiento, también en forma verbal. Muchas veces esto causa confusión. Por una parte la utilización de lenguaje técnico médico el cual no es de fácil entendimiento por el común de las personas, por otra parte por la tensión que un procedimiento quirúrgico produce a las personas o por el poco tiempo que se tiene para dedicarle a dichas explicaciones. Además la información verbal solo le llega a la persona que asistió a la institución de salud, la cual es casi siempre distorsionada al ser trasmitida a su familia. Tampoco queda ninguna constancia de lo que realmente dijo el médico.

Cuando se utiliza material informativo escrito, se da la oportunidad que se lea con tranquilidad en la casa, en compañía de los familiares más cercanos y que se puedan hacer preguntas, las cuales pueden ser resueltas previamente a la cirugía, además queda una constancia de la información del procedimiento y de los riesgos, que fueron explicados.

Existe un material educativo publicado por la sociedad americana de fertilidad (3) y en casi todas las instituciones norteamericanas y europeas, existen dichos materiales, escritos (4) o en videocasetes, pero están poco generalizados en nuestro país.

El material que presento, se ha utilizado durante 1 año y modificado de acuerdo con las preguntas de las usuarias, ver anexo No. 1.

\section{Consentimiento}

En la legislación Colombiana, en el artículo 15 de la Ley 23 de 1981, donde se consagran las actividades y 
advertencia que el médico debe cumplir, se legisla que el médico "Pedirá su consentimiento para aplicar los tratamientos médicos y quirúrgicos que consideren indispensable y que puedan afectarlo física o síquicamente" (2) y en el decreto 3380 de 1981 (5) en el artículo No. 12, se legisla: "El médico dejará constancia en la historia clínica del hecho de la advertencia del riesgo previsto o de la imposibilidad de hacerla".

En la mayoría de nuestras instituciones, la advertencia de riesgos y la autorización para la histeroscopia, se hace en forma verbal y muchas veces no se hace. En algunas instituciones existen formatos de consentimiento o de autorización generales, firmadas al ingreso, en las cuales no se consignan los riesgos específicos del procedimiento, por la cual no tienen ninguna validez legal.

En el anexo No. 2, presento el formato de autorización o consentimiento que he utilizado desde hace un año, el cual fue revisado y aprobado previamente, desde el punto de vista jurídico por la abogada Dra. Bertha Lucy Ceballos.

\section{Informe}

A pesar de los nuevos avances en fotodocumentación y en el desarrollo de videotecas para los procedimientos filmados, no es práctico anexar a todas las historias clínicas estos documentos, por lo cual un buen reporte de la histeroscopia, sigue siendo el método más efectivo y seguro para recordar las observaciones encontradas en una histeroscopia, mas aun, cuando muchos ginecólogos, tratarán a las pacientes, basados únicamente en el informe escrito (6). De aquí la importancia de un informe escrito, completo y exacto.

Cada escuela e institución tiene un informe de histeroscopia diferente.

Recientemente, la Sociedad Colombiana de Obstetricia y Ginecología (6), publicó un formato que se encuentra al final del informe de cirugía endoscópica ginecológica, en el cual no se le da individualidad al informe histeroscópico, disminuyendo la importancia que éste debe tener. El espacio empleado es muy reducido. No hay donde reportar antecedentes de importancia para el histeroscopista, como tamaño uterino, aspecto de la cerviz, histerosalpingografía. El aspecto del exocerviz, la dilatación cervical, pre-operatoria (laminex) o intraoperatoria, el tipo de anestesia utilizado, el medio utilizado para distender la cavidad uterina, ni la cantidad utilizada, de importancia en el post-operatorio, en caso de alguna complicación. No hay donde reportar si se tomó biopsia y su resultado, ni si la visualización de la cavidad fue completa o no. No hay donde consignar un plan o conducta derivado del procedimiento, diagnóstico, ni las complicaciones presentadas, durante o después del procedimiento.
En el anexo No. 3, se encuentra el informe de histeroscopia que utilizo desde hace un año y en el cual ofrezco las siguientes ventajas:

1. Se puede consignar rápidamente los datos de importancia por estar distribuido en forma gráfica, y en algunos casos, solamente marcando con una $x$.

2. Hay espacio para consignar algunos antecedentes de importancia, y algunos hallazgos clínicos, como el tamaño uterino, posición del útero, aspecto de la cerviz, además la dilatación de la cerviz, pre-operatoria con laminex o similar o durante la histeroscopia, de importancia para el manejo posterior o en caso de presentarse complicaciones posteriores.

3. Hay lugar para especificar la indicación de la histeroscopia y los hallazgos previos encontrados en la histerosalpingografía, cuando ésta existe, de importancia para orientar al endoscopista en su búsqueda.

4. Hay sitio para consignar la duración del procedimiento, la utilización y el tipo de anestesia, el nombre del anestesiólogo, el tipo y la cantidad del medio utilizado para distender la cavidad.

5. Los hallazgos se pueden consignar mas gráficamente y quien los ve, puede localizar mas rápidamente el problema reportado por el endoscopista. Y el sitio del cual se tomó biopsia, cuando esta fue aceptada.

6. Hay lugar para reportar si se tomó biopsia y el resultado patológico.

7. Hay lugar para consignar el plan a seguir o la conducta sugerida, derivada del procedimiento endoscópico.

En el informe que planteo, hay un máximo de información, distribuida en una forma gráfica, fácil de llenar y de fácil lectura y comprensión por otros ginecólogos que necesiten del resultado de este examen para determinar tratamientos o conductas.

\section{Conclusiones}

Es necesario difundir modelos educativos previos a los diferentes procedimientos del área médico-quirúrgico, como también modelos de consentimientos o autorizaciones, sugeridos por la legislación, además difundir modelos unificados de informes diagnósticos.

En este artículo, presenté, los tres modelos que estoy utilizando, con el objetivo de proponer su difusión y servir de estímulo para unificar el modelo de informe de los hallazgos encontrados en la histeroscopia, en este momento, en el cual, éste método sé está generalizando en diferentes regiones del país. De esta forma se permitirá más tarde, con mas facilidad, el análisis comparativo de los resultados, no solo entre diferentes instituciones, sino también entre diferentes escuelas de medicina y diferentes regiones, similar al informe de cirugía endoscópica planteado por la Sociedad Colombiana de Obstetricia y Ginecología.

\section{BIBLIOGRAFIA}

1. Azziz R., Murphy AA. "Practical Manual of Operative Laparoscopy and Hysteroscopy" 1992; 151.

2. Código de Etica Médica, Ley 23 de 1981. Artículo 15.

3. The American Fertility Society. "Laparoscopy and histeroscopy, a guide for patients" 1991; 6-9.
4. Dan C. Martin, MD. "Manual of Endoscopy", 1990; 165-167.

5. Código de Etica Medica, decreto 3380 de 1981, artículos 10-12.

6. Argüiello R, et al. Informe de endoscopia ginecológica. Revista Colombiana de Obstetricia y Ginecología. 1997; 48: 119-123. 


\section{HISTEROSCOPIA \\ INFORMACION PARA EL PACIENTE \\ ALBA LUCIA MONDRAGON \\ MEDICINA DE LA REPRODUCCION}

\section{IO QUE USTED DEBE SABER \\ HISTEROSCOPIA} AGERGA DE LA HISTEROSEOPIA

\section{EL UTERO Y CERVIX}

EL UTERO tiene forma de pera, en su parte baja se comunica con la vejiga por una parte angosta llamada cervix, que tiene una abertura en el centro.

\section{DURANTE UN EXAMEN} GINECOLOGICO se puede sentir el tamaño y la posición del útero y ver la abertura del cervix atravez de la vagina, cuando se coloca un especulo.

\section{MIRAR ADENTRO}

Los médicos han buscado por cerca a una centuria, un método simple, seguro y efectivo para mirar adentro de las cavidades de los órganos, pues anormalidades allí adentro no pueden ser encontradas en el examen físico.

Ahora con lentes especiales, con fibras ópticas llamados endoscopios, se puede llevar la luz y permitir que el medico pueda ver el interior de algunos órganos. Por ejemplo : un cistoscopio, es usado para mirar dentro de la vejiga, un proctoscopio, dentro del recto, un colonoscopio, dentro del intestino grueso y un histeroscopio dentro de la cavidad uterina.

\author{
Tiene un especial valor para mujeres \\ que tienen sangrado uteriñ anormal, \\ para que el medico pueda ver la causa \\ de dicho sangrado y en algumas \\ mujeres con infertilidad donde se \\ pueden encontrar insospechados \\ polipos o adherencias. \\ Es una cirugía menor, generalmente libre de \\ complicaciones. No son hechas incisiones y \\ la recuperación es muy corta. \\ Los hallazgos son reconocidos \\ inmediatamente, asi la ansiedad del \\ paciente es rápidamente \\ calmada.
}

ESTE DOCUMENTO DESCRIBE EL PROCEDIMIENTO, PERO POR FAVOR HÁGAME TODAS LAS PREGUNTAS ALREDEDOR DE ESTO, SI NO QUEDAN CONTESTADAS EN LAS SIGUIENTES PAGINAS

\section{GOMO SE REALIZA}

Se puede realizar en el consultorio, no se requieren estudios especiales, preequirurgicos o medicaciones. Se puede hacer en cualquier momento del ciclo menstrual, menos durante la menstruación. El examen no puede ser hecho cuando la paciente tiene un sangrado activo porque la visión es oscurecida. Tampoco se debe hacer cuando hay embarazo o alguna infección vaginal.

La paciente es colocada en la mesa con las piernas elevadas. Se hace un examen físico pélvico para localizar la posición del cervix y del utero. Se coloca un especulo convencional, para exponer el cervix, en el cual se coloca un tenaculo, para mantener esta posición. Algunos pacientes necesitan anestesia 
local inyectable, dependiendo de la necesidad de amortiguar el dolor, pero la mayor no necesitan medicación.

Cuando se va a comenzar a realizar la histeroscopia, la luz es prendida, con el fin de ver la cavidad, las paredes uterinas, deben ser empujadas, para esto se utiliza gas o liquido, La paciente puede experimentar algún cólico mestual, cuando el gas o liquido expande la cavidad uterina.

El extremo del histeroscopio es insertado en el canal cervical y gradualmente dentro de la cavidad uterina. Este instrumento tiene el diámetro de un lápiz y no mas de dos pulgadas entran en la cavidad uterina.

El medico luego, manipula el histeroscopio hasta que el fondo, los lados y los cuernos de la cavidad uterina sean explorados. El proceso de mirar no requiere mas que unos pocos minutos.

\section{DESPUES DEL EXKMIEN}

\author{
Después que el histeroscopio y los otros \\ instrumentos son removidos, algunas \\ pacientes experimentan cólicos, \\ abdominales, 0 dolor de hombros, porque el \\ gas o liquido pasa a través de las trompas, \\ pero esta sensación se va, en pocas horas. La \\ mujer puede salir del consultorio por sus \\ propios medios y no requiere un cuidado \\ post-quirurgico. No hay necesidad de \\ interrumpir la agenda de trabajo. Sangrado \\ menstrual es poco común, pero se puede \\ presentar manchado por unos pocos días.
}

Algunas mujeres tienen un examen histeroscopico en el hospital porque la histeroscopia del consultorio revela algunas anormalidades en la cavidad uterina y se necesita hacer confirmación del diagnostico y también tratamiento al mismo tiempo. Estos tratamientos, en pacientes seleccionadas, pueden aholir la necesidad de cirugía mayor, por ejemplo : adherencias intrauterinas, pueden ser cortadas y removidas, pequeños pólipos y miomas, pueden ser removidos y divisiones anormales del útero, pueden ser corregidos.
Ahora los ginecólogos pueden
mirar cuidadosamente $y$
enteramente toda la cavidad
uterina, encontrando así la causa
de sangrados,

mas certeramente que con un legrado y en algunas situaciones el legrado es innecesario, porque se encuentra una histeroscopia normal.

\section{RIESGOS}

Ninguna cirugía es libre de complicaciones. La posibilidad de perforación uterina, es una complicación que puede ocurrir en 1 de cada 500 exámenes. Esto raramente requiere tratamiento, solamente observación por unas horas.

También pueden presentarse infecciones pélvicas.

Los signos de alarma incluyen:

1. Sangrado abundante

2. Dolor persistente.

3. Fiebre.

Estos síntomas indican un potencial problema y si alguno de ellos ocurre, por favor llámame a mi consultorio y hable conmigo.

Estos síntomas son extremadamente raros.

\section{ULTIMA PALABRA}

La histeroscopia es un significante adelanto en la practica de la ginecología. ES LOGICO MIRAR DENTRO DE LA CAVIDAD UTERINA PARA QUE LAS CAUSAS DE DESORDENES GINECOLOGICOS PUEDAN SER EVIDENTES.

Muchos ginecólogos están aprendiendo este importante procedimiento como un primer paso en el consultorio, con facilidad de paciente ambulatorio y así la frecuente hospitalización puede ser evitada. 


\section{ANEXO NO. 2 \\ AUTORIZACION PARA \\ HISTEROSCOPIA \\ ALBA LUCIA MONDRAGON \\ MEDICINA DE LA REPRODUCCION}

Por medio de la presente yo

con c.c. No.

de

en forma

voluntaria y sin ninguna presión o inducción, consiento en que la Dra. Alba Lucia Mondragón como Cirujana y el

Dr. como anestesiólogo me realicen

histeroscopia, acepto sus riesgos e imprevistos.

Estoy en pleno conocimiento de haber leído ellocumento: HISTEROSCOPIA

información para el paciente, referente a mi próxima cirugia, entiendo lo que he leíc y he discutido ampliamente dicho procedimiento. Además la Dra. Mondragón me ha explicado verbalmente y por escrito, sobre el procedimiento, los cuidados que debo tener antes y después y los riesgos justificados y previsibles del procedimiento quirúrgico que aquí autorizo. También me ha dado la oportunidad de preguntar y resolver las dudas.

Entiendo que la operación puede trae efectos adversos, cambios, imprevistos y complicaciones tales como : ruptura uterina, infección y sangrado y complicaciones anestésicas como : cambios en el ritmo cardiaco, coma y muerte, todas propias del procedimiento que aquí autorizo, las cuales asumo bajo mi propia responsabilidad.

FIRMA DE LA PACIENTE NOMBRE

FIRMA DEL ESPOSO, COMPAÑERO

OTESTIGO

NOMBRE

Este documento esta sujeto a la reglamentación de derechos dẹutor, cualquier reproducción total o parcial, serápenalizada. 


\section{ANEXO NO 3 INFORME DE HISTEROSCOPIA \\ ALBA LUCIA MONDRAGON \\ MEDICINA DE LA REPRODUCCION}

\section{NOMBRE}

FECHA

$\square_{\mathbf{P}} \square_{\mathbf{A}} \square_{\mathbf{C}} \square$

DIA DEL CICLO

CICLOS : REGULARES $O$ IRREGULARES $\bigcirc$

DILATACION : SIO NO O

ANTICONCEPCION : SIO NOO
REMITIDO POR

POSICION UTERO

TAMAÑo UTERINo : NORMAL $\bigcirc$ GRANDE 0 ASPECTO CERVIX

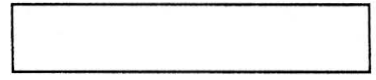

LAMINEX : SI O NO O

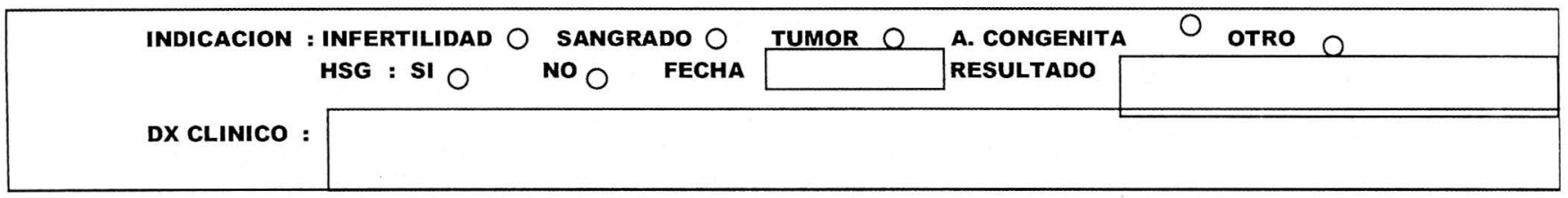

ENDOSCOPISTA

DURACION

MEDIO DISTENCION
ANESTESIOLOGO

ANESTESIA : SI NQ THPO CANTIDAD

\section{HALLAZGOS}

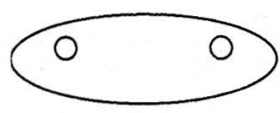

CUERNO OSTIUM DER O DER O IZQ $O$ IZQ $O$

D

A

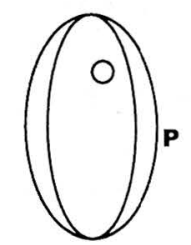

PAREDES
CANAL OCE O OCI $\bigcirc$ ENDOCERVICAL.

ENGROSADO $O$ POLIPOIDEO $O$ HEMORRAGICO O DIVERTICULAR O

ASPECTO DEL ENDOMETRIO : IRREGULAR O DELGADO REGULAR O ENCEFALOIDEO TOMA DE BIOPSIA SI $O$ NO $O$ VISUALIZACION DE LA CAVIDAD COMPLETA SI NO 0

RESULTADO BIOPSIA DIAGNOSTICO ENDOSCOPICO

COMPLICACIONES : NO O SI CONDUCTA POST QX 